\title{
Fast Detection of Image Forgery using Three Step Search Algorithm based on Discrete Cosine Transform
}

\author{
Yong-Dal Shin \\ Dept. of IT \& Securities, U1 (Uone) University \\ ydshin@yd.ac.kr
}

\begin{abstract}
We proposed a fast forgery image detection method using three step search based on discrete cosine transform. We proposed a new three step search based on discrete cosine transform, block distortion measure (BDM), and matching criterion (MC). Our algorithm reduced $99.27 \%$ computational complexity compared to conventional exhaustive search copy move image forgery methods.
\end{abstract}

Keywords: Copy Move Image Forgery, Discrete Cosine Transform, Three Step Search Algorithm

\section{Introduction}

Recently, advanced photo editing software like Photoshop, Paintshop Pro and digital cameras make it relatively easy to create digital forgery images [1]-[12].

Various techniques for tamper images or forgery images detection have been proposed in the literature [1]-[12]. A form of digital forgery is copy-move image forgery. Copy- move image forgery refers to copying a specific area of an image itself and pasting it into another area of the same image [1] - [12]. An example of a forgery image is shown in Figure 1(b). The Figure 1(a) is the original image of lake test image. The Figure 1(b) is a copy-move forgery image [1]-[12].

J. Fridrich [1] proposed an exact match method of forgery image detection for copymoved forgery image. A principal component analysis (PCA) was proposed by A. C. Popescu [2] in order to reduce dimension representation on small size image blocks [9][12]. Most methods of copy-move forgery image detection are divided into overlapping blocks in the searching area [1]-[12]. It is assumed that a test image data of size $\mathrm{N} \times \mathrm{N}$ is divided into $(\mathrm{N}-\mathrm{B}+1)^{2}$ overlapping blocks having a block size of $\mathrm{B} \times \mathrm{B}$ [6], [9] - [12].

However, the exhaustive search method of the copy-move forgery image detection needs huge computational complexity [1]-[4],[9]-[12]. Shin [9]-[12] proposed a fast detection method of the copy-move forgery image using three step search in the spatial domain [10]. When compared to the frequency domain algorithms, the exhaustive search algorithm [1][12], the suggested algorithm reduced the computational complexity. There are various digital watermarking methods as well as copy-move forgery images in image and video forensic methods [13]-[24].

In this paper, we proposed a fast forgery image detection method using three step search (TSS) based on discrete cosine transform (DCT). We proposed block distortion measure (BDM), matching criterion (MC). Our algorithm reduced computational 99.27\% complexity as compared to conventional exhaustive copy-move image forgery method.

Received (December 11, 2017), Review Result (March 8, 2018), Accepted (March 12, 2018) 


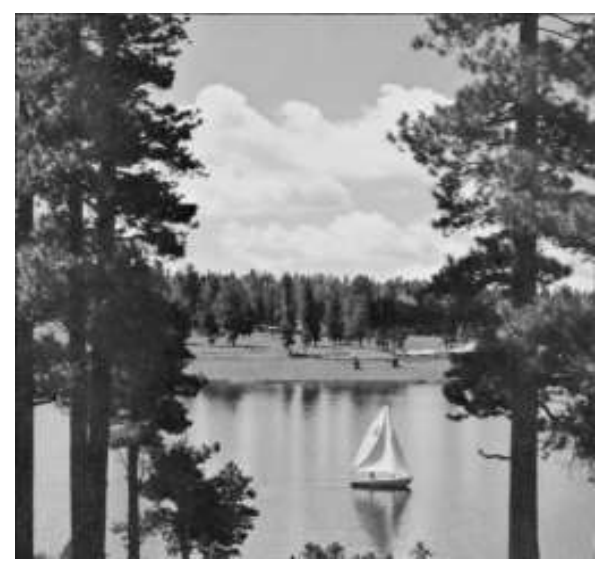

(A) Original Image

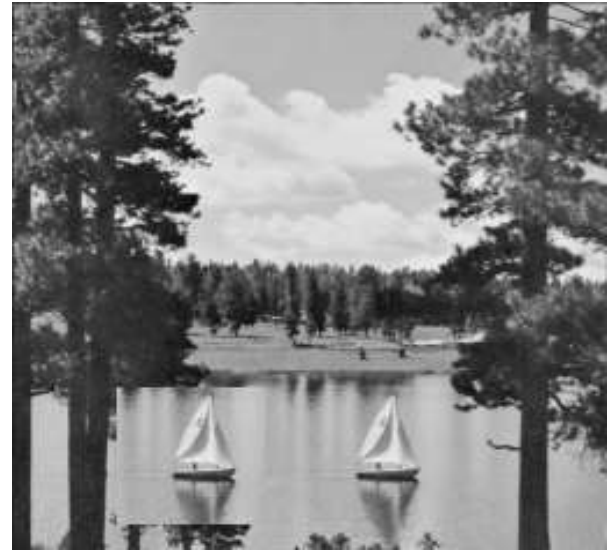

(B) Copy-Move Forgery Image

\section{Figure 1. Lake Test Image}

\section{The Proposed Method}

We proposed a fast forgery image detection method using three step search algorithm based on DCT (TSS-DCT) to reduce computational complexity. The Koga et al., [25] proposed three step search algorithm in the spatial domain [9]-[12]. It became very popular in the motion estimation for moving pictures because of its simplicity and near optimal performance. The algorithm is described in [25]. We proposed three step search algorithm using DCT. Our TSS-DCT algorithm works as follows. The DCT is calculated by using $8 \times 8$ pixel blocks. We obtained DCT coefficients of $8 \times 8$ pixel block in the frequency domain. The DCT algorithm [25] transforms the image signal of the spatial domain into that of the frequency domain $[9-12,25]$. Most of image signal energy lies in the low frequency region [25]; these appear DCT four $(0,1,2,3)$ coefficients of Figure $2[10,25]$. The higher frequencies are often small values $[7,10]$, so the higher frequency areas can be neglected with little visible distortion [7, 10]. DCT transform algorithm is equation (1). The Inverse DCT algorithm is shown in Equation (2) [25].

$$
\mathrm{X}(\mathrm{u}, \mathrm{v})=\frac{2}{B} \mathrm{C}(\mathrm{u}) \mathrm{C}(\mathrm{v}) \sum_{i=0}^{B-1} \sum_{j=0}^{B-1} x(i, j) \cos \left[\frac{(2 i+1) u \pi}{2 B}\right] \cos \left[\frac{(2 j+1) v \pi}{2 B}\right]
$$

Inverse DCT (IDCT) is

$$
\begin{aligned}
\mathrm{x}(\mathrm{i}, \mathrm{j}) & =\frac{2}{B} \mathrm{C}(\mathrm{u}) \mathrm{C}(\mathrm{v}) \sum_{u=0}^{B-1} \quad \sum_{v=0}^{B-1} X(u, v) \cos \left[\frac{(2 i+1) u \pi}{2 B}\right] \cos \left[\frac{(2 j+1) v \pi}{2 B}\right] \\
\mathrm{C}(0)=\frac{1}{\sqrt{2}}, \mathrm{C}(\mathrm{u}) & =\mathrm{C}(\mathrm{v})=1 \quad(\mathrm{u} \neq 0, \mathrm{v} \neq 0), \text { Where, } \mathrm{B} \text { is a block size }
\end{aligned}
$$

The Transform coefficients for $\mathrm{U}=0, \mathrm{DC}$, is the average value of the $\mathrm{B} \times \mathrm{B}$ pixel block, where $B$ is $8 \times 8$ pixels $[10,26]$. All other coefficients are the AC coefficients except $U=0$ DC value $[10,25]$.

The algorithm try to find copy-moved motion vectors for forgery image [9-12]. The copy moved motion vectors can be obtained by using TSS-DCT, which reduces computational complexity. In the following, we describe the proposed algorithm in detail.

Step 1: We compute DCT coefficients for $8 \times 8$ pixel block of input image [10, 25]. The TSS-DCT algorithm begins with DCT coefficients locations at the center and sets the step size (SS) SS=4, 4-pixel/4-line (+/- 4) [10, 26] as shown in Figure 2. It then searches eight DCT coefficients locations $+/-$ SS pixels in the $\mathrm{x}$ and $\mathrm{y}$ direction of DCT coefficients 
location $(0,0)$ as well as the center location [10],[26]] as shown in Figure 2.

Step 2: The locations retrieved from these nine DCT coefficient locations are chosen to provide the lowest cost and are made into a new search origin $(4,0)$ as shown in Figure 1. The second step starts with a new step size of SS = SS / 2 = 2, 2-pixel / 2-line [10], and [26] centered on the DCT coefficient of the selected position in the first step, and thus, we find the best matching value, $(2,-2)$ as shown in Figure $2[10,26]$.

Step 3: The third step searches based on 1-pixel/1-line DCT coefficients resolution [10, 26] and i.e., $3 \times 3$ DCT coefficients search window around the location determined at the second step $[10,26]$. The best-match block in the three step is found. The DCT coefficients location $(1,-3)$ is the copy moved motion vector value $[10,26]$.

The computational complexity of TSS algorithm needs 25 DCT coefficients checking points. In the case of a maximum displacement window of 7 i.e., $\mathrm{w}= \pm 7[10,26]$, the total number of checking points required is $[9($ Step 1$)+8($ Step 2$)+8($ Step 3$)]$ or $25[10,26]$ for search window $15 \times 15$ DCT coefficients. On the other hand that of exhaustive search check points are $225[10,26]$.

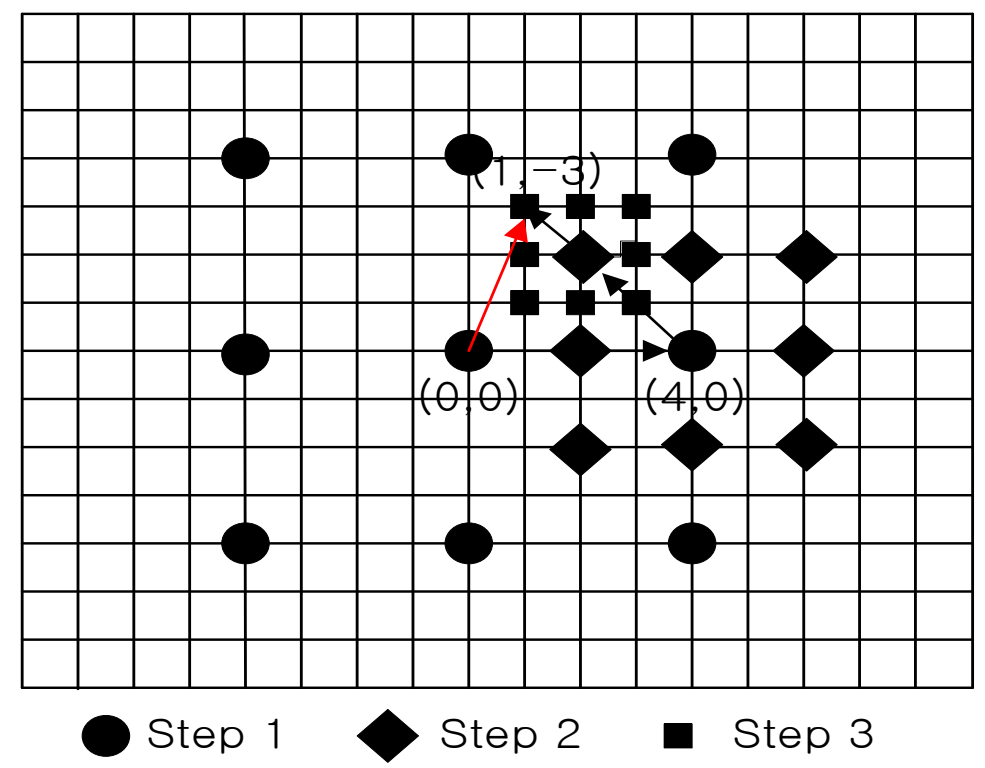

\section{Figure 2. Three Step Search Algorithm using DCT Domain (Motion Vector is $(1,-3)$ )}

In order to find copy moved image forgery motion vectors $\mathrm{x}$ and $\mathrm{y}$, we calculated minimum of BDM of eq. (5) using TSS-DCT. We used block distortion measure (BDM) for matching criterion of copy-moved forgery image detection using TSS-DCT in this paper. The BDM can be expressed as in Equation (3).

$$
B D M=\sum|F I(i+i i, j+j j)-F I(i+i i+y, j+j j+x)|
$$

where, FI (i+ii, j+jj) is DCT coefficients of $8 \times 8$ pixel block at the position $(i, j)$ of reference region image. $i=0,1,2,3 \ldots . \mathrm{N}-\mathrm{B}+1, j=0,1,2,3 \ldots \mathrm{N}-\mathrm{B}+1 . \mathrm{ii}, \mathrm{jj}=0,1 . \mathrm{FI}(\mathrm{i}+\mathrm{ii}+\mathrm{x}, \mathrm{j}+$ $\mathrm{jj}+\mathrm{y})$ is DCT coefficients of $8 \times 8$ pixel block at position $(i+i i, j+j j)$ of the matching search region image. $\mathrm{x}$ and $\mathrm{y}$ are motion vectors obtained by TSS-DCT. The maximum displacement window is 7 for DCT-TSS. The size of test images is $256 \times 256$ pixels. The block size of DCT is $8 \times 8$ pixel block. The TSS-DCT is divided into $15 \times 15$ pixel blocks 
non-overlapping DCT coefficients pixel block in the matching search region image. Thus, the computational complexity 64 is reduced by 4 in the $8 \times 8$ pixel block to obtain motion vector $\mathrm{x}, \mathrm{y}$ in the TSS-DCT. The ii and jj of Equation (3) need 4-computational complexity, which is shown in 0,1,2,3 of Figure 3. Hence, the proposed BDM of TSS-DCT reduces computational complexity.

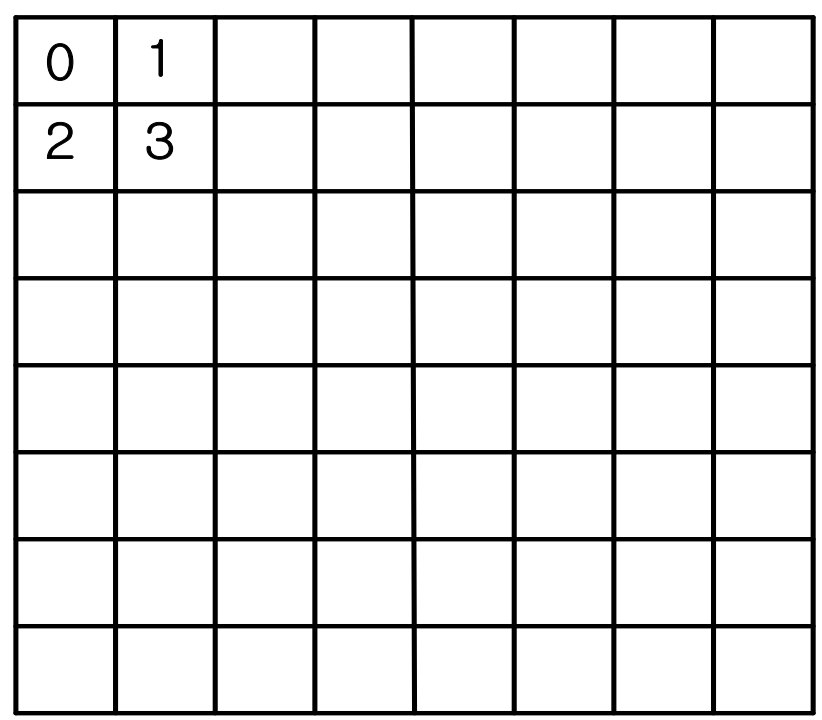

Figure 3. TSS-DCT four $(0,1,2,3)$ Low Frequency Coefficients for $8 \times 8$ Pixel Block

The matching criterion (MC) of copy-moved image forgery detection using DCT coefficients for block image as follow.

After motion vectors $\mathrm{x}$ and $\mathrm{y}$ are obtained from Equation (3), $\mathrm{ii}$ and $\mathrm{jj}=0,1,2 \ldots . \mathrm{B}-1$ of equation (3) is used to detect copy-moved image forgery block. The MC is matching criterion used to detect copy moved image forgery using TSS-DCT algorithm.

$$
\mathrm{MC}=\sum|\mathrm{FI}(\mathrm{i}+\mathrm{ii}, \mathrm{j}+\mathrm{jj})-\mathrm{FI}(\mathrm{i}+\mathrm{ii}+\mathrm{y}, \mathrm{j}+\mathrm{jj}+\mathrm{x})|
$$

where FI ( $\mathrm{i}+\mathrm{ii}, \mathrm{j}+\mathrm{jj})$ is DCT coefficients of $8 \times 8$ pixel block at the position $(\mathrm{i}, \mathrm{j})$ of reference region image. $i=0,1,2,3 \ldots \mathrm{N}-\mathrm{B}+1, \mathrm{j}=0,1,2,3 \ldots \mathrm{N}-\mathrm{B}+1$. ii and $\mathrm{jj}=0,1 \ldots \mathrm{B}-1$. $\mathrm{FI}(\mathrm{i}+\mathrm{ii}+\mathrm{x}, \mathrm{j}+\mathrm{jj}+\mathrm{y})$ is DCT coefficients of $8 \times 8$ pixel block at position $(\mathrm{i}+\mathrm{ii}, \mathrm{j}+\mathrm{jj})$ of the matching search region image. $\mathrm{x}$ and $\mathrm{y}$ are motion vectors.

The MC is a matching criterion of copy-moved image forgery detection. The MC is summation of difference between gray level of reference image block and matching search image block. We used matching criterion to detect copy-moved image forgery block. The detection method of the copy moved image forgery based on matching criterion is expressed by eq. (5). If the feature vectors of two blocks are coincide, they would give same DCT coefficients.

If $(\mathrm{MC}==0)$

Copy-moved image forgery block

Else

Not copy-moved image forgery block 
The proposed method consisted of three step search algorithm based on DCT frequency domain, BDM, MC, and detection method. From equation (5), if the MC were 0, the block is a copy-moved image forgery block [9-11]. If the $\mathrm{MC}$ were not 0 , the block image is not copy-move forgery block [9-11]. This indicates that the copied block image is different from the pasted block image [9-11]. The Figure 4 is shown by the flow chart of proposed method

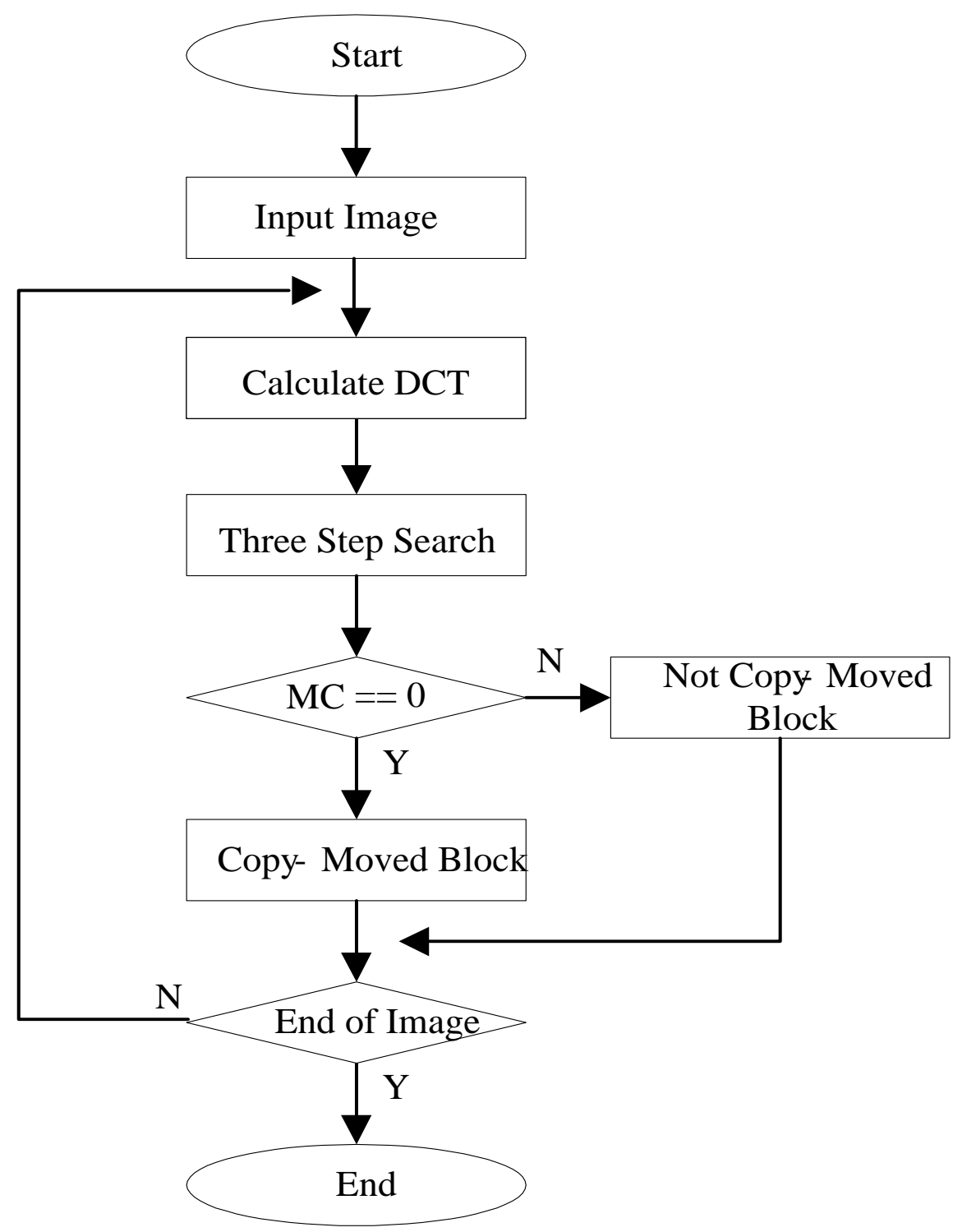

Figure 4. The Flow Chart of Proposed Method using TSS Algorithm

\section{Experimental Results}

We proposed a new fast detection method for copy-moved image forgery using TSSDCT in the DCT frequency domain. The proposed TSS-DCT algorithm reduced computational complexity as compared to various conventional methods [9-12].

The test images used to perform experiments Car3, Bridge, House, Lake, and Airplane images. Their gray scales is 8 bits and resolution is $256 \times 256$ pixels [9]-[12],[27]. The matching search region of test images are divide into $15 \times 15$ (TSS-DCT) $f$ non-overlapping 
from first image location $(0,0)$ to last image location $(255,255)$ [9]-[11]. The MC of copymoved image forgery detection is Equation (6) based on TSS-DCT algorithm.

In this paper, the computational complexity of TSS-DCT algorithm needs 4 DCT coefficients of TSS-DCT per checking point for $15 \times 15$ pixel block. The BDM of TSS-DCT algorithm is sufficient by 4 low frequency DCT coefficients per checking point instead of 64 checking points (8x8 pixel block search complexity) to reduce computational complexity.

The Table 1 shows computational complexity results of the proposed TSS-DCT algorithm and conventional copy move methods for test 5 images base on reference [1]. The proposed TSS-DCT algorithm 100\% detected copy-move forgery image perfectly.

From Table 1, IR, BS, MSRBN, FD, CC are image representation, reference region block size, matching search region block number, feature dimension, computation complexity (based on reference [1]) method, respectively.

\section{Table 1. Computational Complexity Results of the Proposed TSS-DCT Algorithm and other Conventional Methods}

\begin{tabular}{cccccl}
\hline Algorithms & IR & BS & MSRBN & FD & CC(\%) \\
\hline Exhaustive & Spatial & $8 \times 8$ & $(256-8+1)^{2}$ & 64 & 100.0 \\
Fridrich[1] & DCT & $8 \times 8$ & $(256-8+1)^{2}$ & 64 & 100.0 \\
Popescu[2] & PCA & $8 \times 8$ & $(256-8+1)^{2}$ & 32 & 50.0 \\
Kahn[7] & DWT & $8 \times 8$ & $(128-8+1)^{2}$ & 64 & 23.61 \\
Proposed & DCT- & $8 \times 8$ & $25 \times 17^{2}$ & 4 & 0.73 \\
algorithm & TSS & & & & \\
\hline
\end{tabular}

The proposed method reduced $99.27 \%$ of computational complexity than exhaustive search by [1]. From Table 1, the proposed TSS-DCT algorithm reduced computational complexity more than conventional various copy move forgery image detection method [1, $2,7]$, because the proposed TSS-DCT algorithm used which the characteristic of DCT compression in the frequency domain, it used 4 low frequency DCT coefficients such discrete cosine transform, also it replace one pixel shift (conventional methods) by nonoverlapping $15 \times 15$ pixel block (TSS-DCT algorithm search region) to detect copy-moved image forgery in matching search region.

The Figure 5, 6, 7, 8, and 9 showed results of proposed method. We showed original images, copy-moved image forgery, detection of copy-moved image forgery in the Figure $5,67,8$, 9. From the Figure 5(c) and 6(c), left black box is copied, right black box is moved to image forgery block. From Figure 7(c), 8(c), and 8(c), right black box is copied, left black box is moved to image forgery block. From Figures 5, 6, 7, 8, and 9 our algorithm detected copy-moved image forgery $100 \%$ perfectly.

\section{Conclusion}

In this paper, we proposed a fast forgery image detection method using three step search (TSS) based on discrete cosine transform (DCT). We proposed block distortion measure (BDM), matching criterion (MC). Our algorithm reduced computational 99.27\% complexity as compared to conventional exhaustive copy-move image forgery method. 


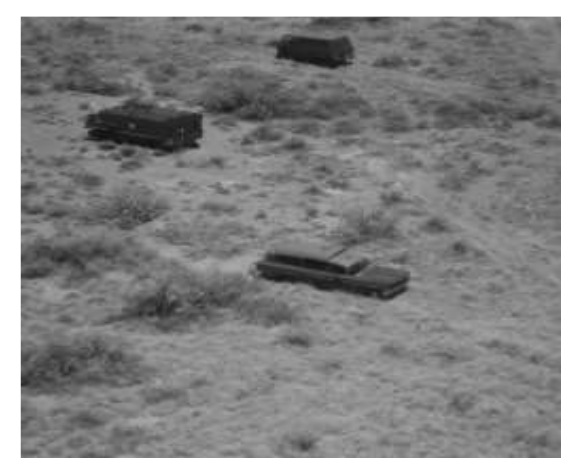

(a) Original Car3 Image

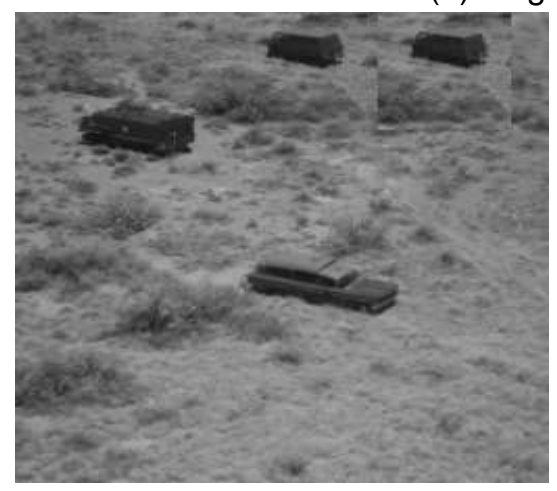

(b) Copy-Moved forgery Car3 Image

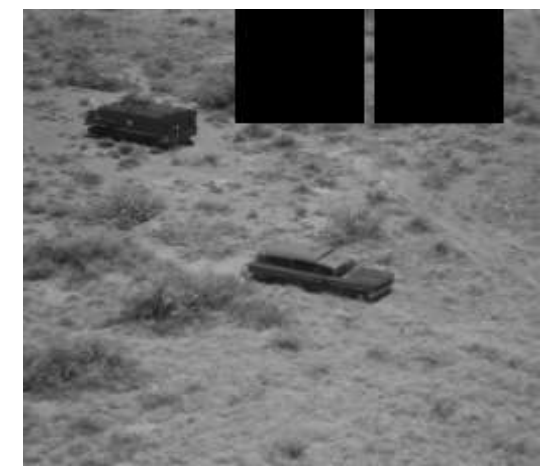

(c) Detection block of Copy- Move Forgery Car3 Image (Black box)

Figure 5. Result of Proposed Method for Car3 Image

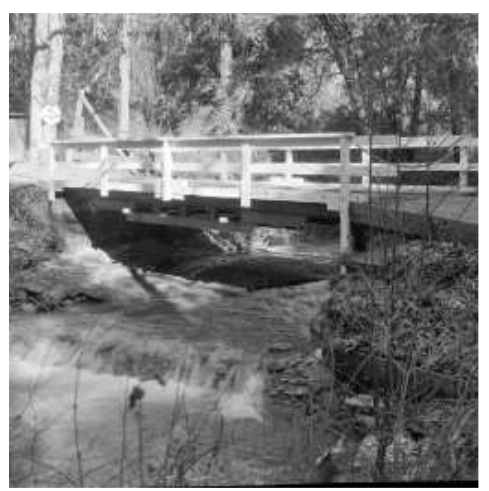

(a) Original Bridge Image

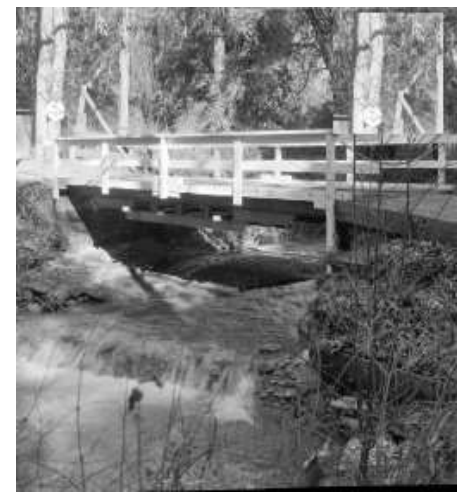

(b) Copy-Moved Forgery Bridge Image

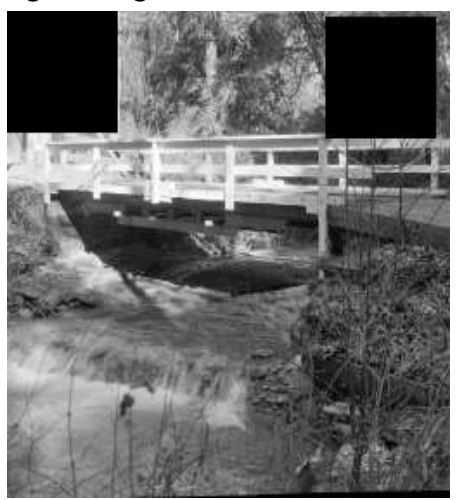

(c) Detection block of Copy- Moved Forgery Bridge Image (Black box)

Figure 6. Result of Proposed Method for Bridge Image 


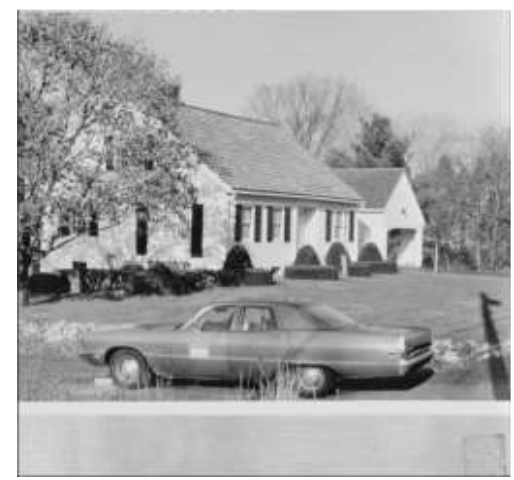

(a) Original House Image

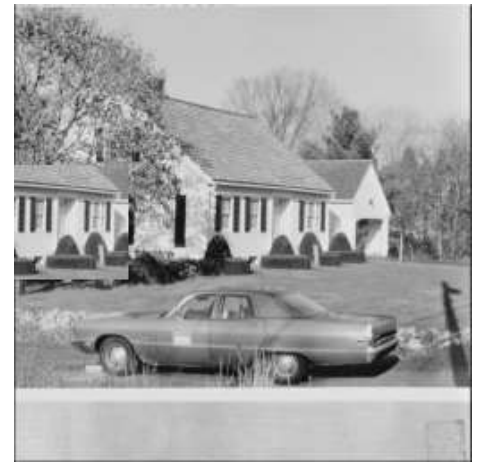

(b) Copy-Moved Forgery House Image

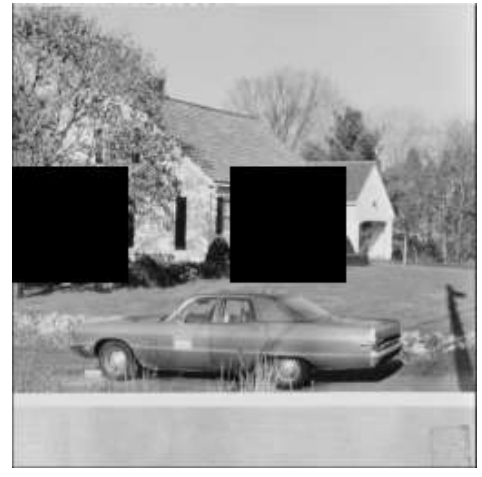

(c) Detection Block of Copy- Moved Forgery House Image (Black box)

Figure 7. Result of Proposed Method for House Image

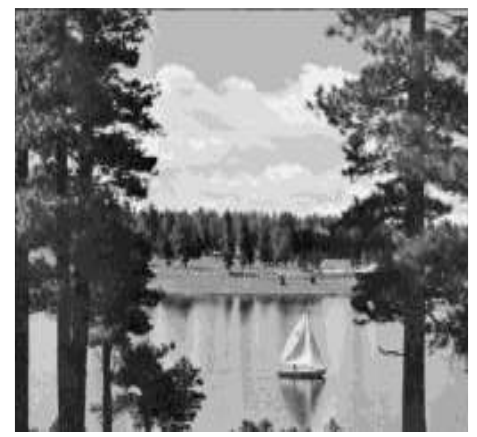

(a) Original Lake Image

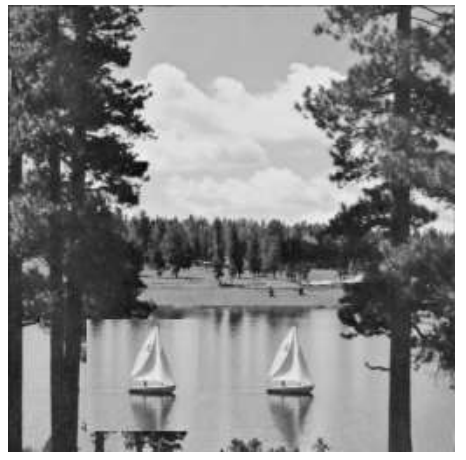

(b) Copy-Moved Forgery Lake Image

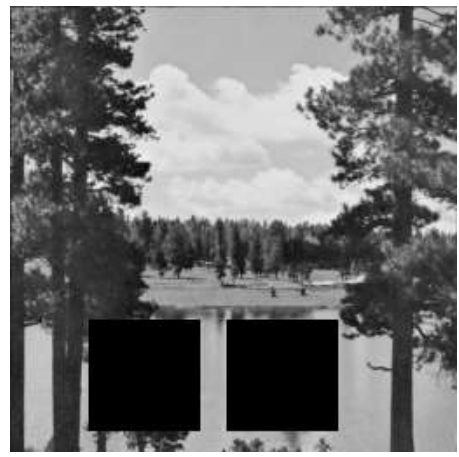

(c) Detection Block of Copy- Moved Forgery Lake Image (Black box)

Figure 8. Result of Proposed Method for Lake Image 


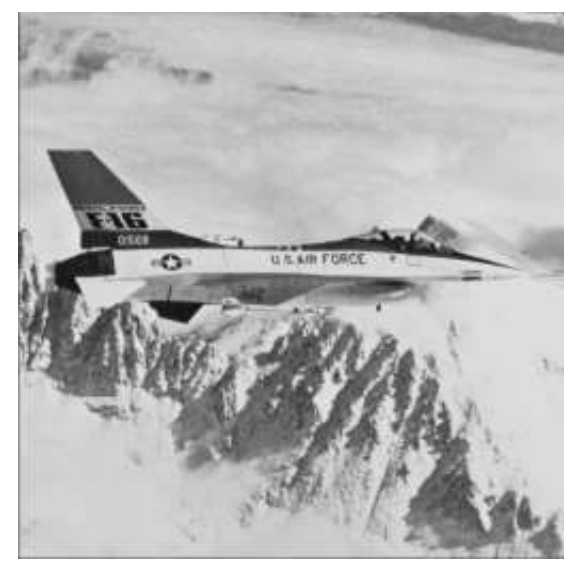

(a) Original Airplane Image

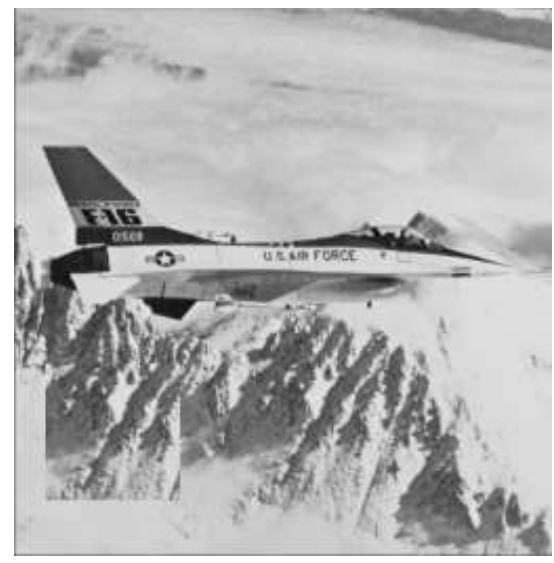

(b) Copy-Moved Forgery Airplane Image

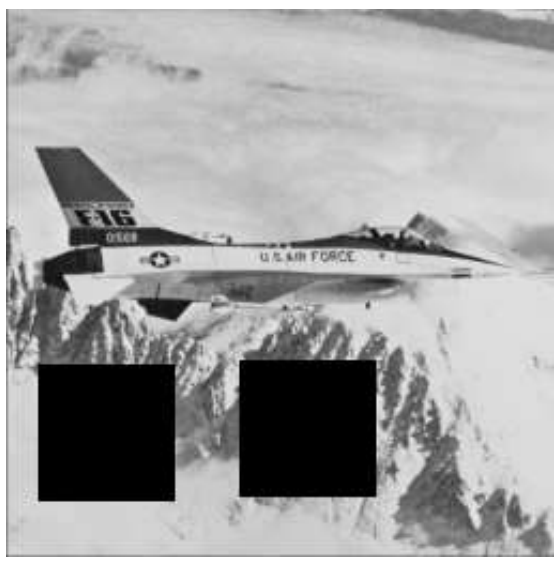

(c) Detection block of Copy- Moved Forgery Airplane Image (Black box)

Figure 9. Result of Proposed Method for Airplane Image

\section{Acknowledgments}

This paper is a revised and expanded version of a paper entitled "Analysis of copy move forgery image detection", in the $13^{\text {th }}$ International Workshop Series, presented at, Jeju, Korea. (2017), Aug. 20-22.

\section{References}

[1] J. Fridrich, D. Soukal and J. Lukas, "Detection of copy-move forgery in digital images", Proceedings of Digital Forensic Research Workshop, (2003), pp. 55-61.

[2] A. C. Popescu and H. Farid, "Exposing digital forgeries by detecting duplicated image regions", Dartmouth College, Hanover, New Hampshire, USA:TR2004-515, (2004).

[3] V. Christlein, C. Riess, J. Jordan and E. Angelopoulou, "An Evaluation of Popular Copy-Move Forgery Detection Approaches", IEEE Transactions on Information Forensics and Security, vol. 7, no. 6, (2012), pp. 1841-1854.

[4] D. Mahalakshmi, S. K. Vijayalakshmi and S. Priyadharsini, "Digital image forgery detection and estimation by exploring basic image manipulations", Digital Investigation, vol. 8, no. 3, (2012), pp. 215225.

[5] E. S. Khan and E. A. Kulkarni, "An efficient method for detection of copy-move forgery using discrete wavelet transform", International Journal of Computer Science and Engineering vol. 2, no. 5, (2010), pp. 1801-1806.

[6] H. J. Lin, C. W. Wang, and Y.T. Kao, "Fast copy-move forgery detection”, WSEAS Transaction on Signal Processing vol. 5, issue 5, (2009), pp. 188-197.

[7] S. Kahn and A. Kulkarni, "Reduced time complexity for detection of copy-move forgery using discrete wavelet transform", International Journal of Computer Applications vol. 6, no. 7, (2010), pp. 31-36. 
[8] J. Casey, "An investigation of block searching algorithms for video frame coders", Dublin Institute of Technology, masters of Science thesis, (2008).

[9] Y. D. Shin, "Fast detection of copy-move forgery image using two step search algorithm", International Journal of Security and Its Applications vol. 10, no. 5, (2016), pp. 203-214.

[10] Y. D. Shin, "Fast detection of copy-move forgery image using three step search algorithm in the spatial domain", Convergence and Hybrid Information Technology, ed: Springer Berlin Heidelberg, Daejeon, Korea, (2012) August 23-25, pp. 389-395.

[11] Y. D. Shin, "Fast detection of copy-move forgery image using DCT", Journal of Korea Multimedia Society, vol. 16, no. 4, (2013), pp. 411-417.

[12] Y. D. Shin, "Analysis of copy move forgery image detection", The $13^{\text {th }}$ International Workshop Series, Jeju, Korea, (2017) August 20-22.

[13] C.-T. Hsieh, Y.-K. Wu and K.-M. Hung, "Hybrid Watermarking Scheme for Halftone Images", International Journal of Advanced Science and Technology, vol. 1, (2008), pp. 9-20.

[14] G. Rosline Nesa Kumari, B. VijayaKumar, L. Sumalatha and Dr. V. V. Krishna, "Secure and Robust Digital Watermarking on Grey Level Images", International Journal of Advanced Science and Technology, vol. 11, (2009), pp. 1-8.

[15] M. Mohamed Sathik and S. S. Sujatha, "An Improved Invisible Watermarking Technique for Image Authentication", International Journal of Advanced Science and Technology, vol. 24, (2010), pp. 61-74.

[16] M. Mohamed Sathik and S. S. Sujatha, "Wavelet Based Blind Technique by Espousing Hankel Matrix for Robust Watermarking", International Journal of Advanced Science and Technology, vol. 26, (2011), pp. 57-72.

[17] Sachin Goyal and R. Gupta, "Optimization of Fidelity with Adaptive Genetic Watermarking Algorithm using Tournament Selection”, International Journal of Advanced Science and Technology, vol. 30, (2011), pp. 55-66.

[18] R. Keshavarzian and A. Aghagolzadeh, "Block and Region Based Image Error Concealment Using Fragile Watermarking in the Spatial Domain", International Journal of Advanced Science and Technology, vol. 46, (2012), pp. 1-16.

[19] Md. I. Hasan Sarker, M. Ibrahim Khan, K. Deb and Md. F. Faruque, "FFT-Based Audio Watermarking Method with a Gray Image for Copyright Protection", International Journal of Advanced Science and Technology, vol. 47, (2012), pp. 65-76.

[20] J. Waleed Salih, S. Hameed Abid and T. Mohammed Hasan, "Imperceptible 3D Video Watermarking Technique Based on Scene Change Detection", International Journal of Advanced Science and Technology, vol. 82, (2015), pp. 11-22.

[21] J. Abraham and V. Paul, "Invisible Image Watermarking on Selected Regions using DWT", International Journal of Advanced Science and Technology, vol. 98, (2017), pp. 46-56.

[22] K. Amudha, C. Nelson Kennady Babu and S. Balu, "Enhancing Information Capacity and Security Level Based on Reversible Watermarking Technique in Medical Images", International Journal of u - and e Service, Science and Technology, vol. 9, no. 7, (2016), pp. 121-130.

[23] C.-C. Chang and H. Chou, "A New Public-key Oblivious Fragile Watermarking for Image Authentication Using Discrete Cosine Transform”, International Journal of Signal Processing, Image Processing and Pattern Recognition, vol. 2, no. 1, (2009), pp. 133-140.

[24] P. U. Lande, S. N. Talbar and G. N. Shinde, "A Fuzzy Logic Approach to Encrypted Watermarking for Still Images in Wavelet Domain on FPGA", International Journal of Signal Processing, Image Processing and Pattern Recognition, vol. 3, no. 2, (2010), pp. 1-10.

[25] P. Yip and K. Rao, "Discrete Cosine Transform", Algorithms, and Applications, Academic press, CA, (1990).

[26] T. Koga, K. Iinuma, A. Hirano, Y. Iijima and T. Ishiguro, "Motion compensated interframe coding for video conferencing", Pro. Nat. Telecommun. Conf., New Orleans, (1981), pp. G5.3.1-5.3.5.

[27] http://sipi.usc.edu/database.

\section{Author}

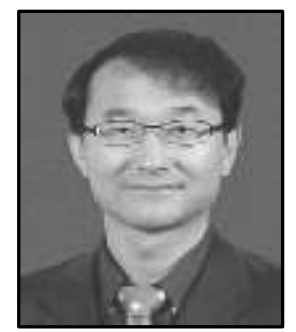

Yong-Dal Shin, is a professor in department of IT \& securities at U1 university, Choongnam Korea. He received Ph.D. degree from Kyungpook national university, Daegu Korea, 1994. He research areas include multimedia security, digital watermarking, digital forensics. 